International Journal of Pure and Applied Mathematics

Volume 97 No. 1 2014, 89-97

ISSN: 1311-8080 (printed version); ISSN: 1314-3395 (on-line version)

url: http://www.ijpam.eu

doi: http://dx.doi.org/10.12732/ijpam.v97i1.9

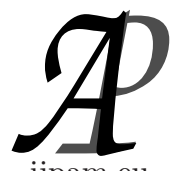

ijpam.eu

\title{
DUAL-TREE COMPLEX WAVELET TRANSFORM APPLIED TO FAULT MONITORING AND IDENTIFICATION IN AERONAUTICAL STRUCTURES
}

\author{
C.C.E. Abreu ${ }^{1}$, F.R. Chavarette ${ }^{2}$, F. Villarreal ${ }^{3}$, \\ M.A.Q. Duarte ${ }^{4}$, F.P.A. Lima ${ }^{5}$ \\ ${ }^{1,5}$ Electrical Engineering PhD Program \\ UNESP - Univ. Estadual Paulista \\ Brasil Avenue, 56, 15385-000, Ilha Solteira, SP, BRAZIL \\ ${ }^{2,3}$ Mathematics Department \\ UNESP - Univ. Estadual Paulista \\ Brasil Avenue, 56, 15385-000, Ilha Solteira, SP, BRAZIL \\ ${ }^{4}$ Universidade Estadual de Mato Grosso do Sul \\ Unidade Universitária de Cassilândia \\ Rodovia MS 306 KM 06, 79540-000 \\ Cassilândia, MS, BRASIL
}

\begin{abstract}
This paper proposes the application of the dual-tree complex wavelet transform on fault monitoring and identification in aeronautical structures. For this purpose, a multiscale analysis from phase of the complex wavelet coefficients is proposed. Phase is very sensitive to variations of the signal capturing small flaws. Furthermore, magnitude provides information about the intensity of failure. Simulation results from a beam model showed that the proposed method achieved good performance in small flaws identification.
\end{abstract}

AMS Subject Classification: $42 \mathrm{C} 40$

Key Words: dual-tree complex wavelet transform, fault monitoring and identification, aeronautical structures

Received: July 18, 2014

(c) 2014 Academic Publications, Ltd.

${ }^{\S}$ Correspondence author url: www.acadpubl.eu 
90 C.C.E. Abreu, F.R. Chavarette, F. Villarreal, M.A.Q. Duarte, F.P.A. Lima

\section{Introduction}

The improvement of the wavelet analysis, specifically the discrete wavelet transform (DWT), enabled the development of new techniques for digital signal processing in several areas. Among them, there are the techniques dedicated for speech enhancement, image enhancement, applications in medicine and in fault monitoring and identification.

In signal processing, the wavelet analysis provides a representation process of a signal by the orthonormal basis functions that oscillate locally, those functions are called wavelets. In other words, wavelets have compact support, unlike the Fourier analysis which is based on infinitely oscillating sinusoidal basis functions.

In the classical sense, we say $\psi(t) \in L^{2}(\mathbb{R})$ is a wavelet if its Fourier transform $\hat{\psi}(\omega)$ satisfies the condition:

$$
C_{\psi}=\int_{-\infty}^{+\infty} \frac{|\hat{\psi}(\omega)|^{2}}{|\omega|} d \omega<\hat{\psi}(\omega)
$$

From (1), it follows that $\lim _{\omega \rightarrow 0} \hat{\psi}(\omega)=0$ [1]. This way, if $\hat{\psi}(\omega)$ is continuos $\hat{\psi}(0)=0$. Since

$$
\hat{\psi}(\omega)=\int_{-\infty}^{+\infty} \psi(t) e^{-j \omega t} d t
$$

it follows that $\int_{-\infty}^{+\infty} \psi(t) d t=0$. In this way, a wavelet must oscillate in order to cancel the positive and negative areas.

A wavelet basis consists of stretched and shifted versions of a fundamental function $\psi$. When carefully combined with the shifted version of a scaling function $\phi$, it is performed a signal expansion through an orthonormal basis. This base provides a time-frequency analysis of the analyzed signal. Therefore, any analog signal $x(t)$ can be expressed in terms of wavelets and scaling functions as follows:

$$
x(t)=\sum_{n=-\infty}^{+\infty} c(n) \phi(t-n)+\sum_{j=0}^{+\infty} \sum_{n=-\infty}^{+\infty} d(j, n) 2^{j / 2} \psi\left(2^{j} t-n\right),
$$

where $c(n)$ and $d(j, n)$ are scaling and wavelet coefficients, respectively. $c(n)$ and $d(j, n)$ are calculated via the inner product [2]:

$$
c(n)=\int_{-\infty}^{+\infty} x(t) \phi(t-n) d t
$$




$$
d(j, n)=2^{j / 2} \int_{-\infty}^{\infty} x(t) \psi\left(2^{j} t-n\right) d t .
$$

Together, (3), (4) and (5) provide a time-frequency analysis controlled by the scale factor $j$ in the time shift $n$.

There exist very efficient algorithms to compute $c(n)$ and $d(j, n)$. Mallat, in [4], proposed an algorithm for applying a digital filter bank in a tree structure. It consists of successive applications of a low-pass/highpass filter pair followed by a dyadic donwsampling. The outputs of the low-pass $\left(h_{0}\right)$ and high-pass $\left(h_{1}\right)$ filters are respectively termed approximation, $c(n)$, and detail $d(j, n)$, coefficients of the signal decomposition at level $j(j=1,2,3, \ldots)$. The filters $h_{0}$ and $h_{1}$ satisfy the perfect reconstruction conditions and are associated with a wavelet function, chosen a priori according to the characteristics of the signal in analysis [1]. This decomposition process is extensively used in order to evaluate the discrete wavelet transform (DWT).

These filters enable the formulation of the parameterization in order to design wavelets and scaling functions with some interesting properties, i.e., compact support and vanishing moments [5]. Furthermore, such architecture provides a time-frequency analysis, in view that $d(j, n)$ correspond to the frequency situated approximately between $\left(2^{-j} f_{s}, 2^{-j-1} f_{s}\right)$, where $f_{s}$ is the sampling rate of the signal $x(t)[3]$.

In spite of the DWT be a powerful tool for studying nonstationary signals, in [2], authors highlight some shortcomings:

- wavelets have bandpass characteristics and their coefficients oscillate positively and negatively around singularities. This fact makes the signal singularities extraction an delicate process.

- DWT is shift variance.

- the lack of directional selectivity in dimensions higher than one, that might be a problem in image processing.

In this sense, algorithms must be projected to be able of coping with such characteristics.

Fortunately, some drawbacks of the DWT are overcome by the complex wavelet transform $(\mathbb{C W T})[2]$. The dual-tree complex wavelet transform (dualtree $\mathbb{C W T}$ ) proposed by Kingsbury in [6] is near shift-invariance, directionally selective in higher dimensions and also provides other desirable properties at small additional computational costs [12]. 
92 C.C.E. Abreu, F.R. Chavarette, F. Villarreal, M.A.Q. Duarte, F.P.A. Lima

This paper shows the advantages in using complex wavelets for fault monitoring and identification in aeronautical Structures. For this purpose, the dualtree architecture will be discussed. The remainder of the paper is organized as follows: The dual-tree complex wavelet transform is presented in Section 2, its applicability in fault identification in aeronautical structures is presented in Section 3. Finally, concluding remarks are presented in Section 4.

\section{Dual-Tree Complex Wavelet Transform}

The dual-tree $\mathbb{C W T}$ was introduced by Kingsbury [6] in 1998 as an enhancement to the DWT: it is nearly shift-invariant and directionally selective in two and higher dimensions [2]. Complex wavelet design takes into account some desirable characteristics of the Fourier transform [2]. Note that the Fourier transform is based on complex-valued oscillating sinusoids as in (6).

$$
e^{j \Omega t}=\cos (\Omega t)+j \sin (\Omega t) .
$$

Real and imaginary parts are $90^{\circ}$ out of phase with each other, thus forming a Hilbert transform pair. According to [2], together they constitute an analytic signal $e^{j \Omega t}$, where $\Omega>0$ and $j=\sqrt{-1}$. Furthermore, the magnitude of the Fourier transform is perfectly shift-invariant [9].

Inspired by the Fourier transform, the dual-tree $\mathbb{C W T}$ is an effective approach for implementing an analytic wavelet transform. The dual-tree $\mathbb{C W T}$ is based on scaling and wavelet functions with complex values, according to (7) and (8).

$$
\begin{aligned}
& \psi_{c}(t)=\psi_{r}(t)+j \psi_{i}(t), \\
& \phi_{c}(t)=\phi_{r}(t)+j \phi_{i}(t) .
\end{aligned}
$$

Similarly to $(6), \psi_{i} \approx \mathcal{H}\left\{\psi_{r}\right\}$. Moreover, $\psi_{r}$ is real and even and $j \psi_{i}$ is imaginary and odd [2]. The complex scaling function is defined similarly. Complex coefficients generated by the dual-tree $\mathbb{C W T}$ can be represented by:

$$
d_{c}(j, n)=d_{r}(j, n)+j d_{i}(j, n) .
$$

Real and imaginary parts $d_{r}(j, n)$ and $d_{i}(j, n)$, respectively, are calculated individually by two filters banks, see Figure 1. In other words, the dual-tree $\mathbb{C W T}$ employs two discrete wavelet transforms, where the upper tree produces the real part and the lower tree produces the imaginary part of the complex coefficients. Both sets of filters are designed such as the overall transform is approximately analytic and near shift-invariant [6]. 
In Figure 1, $h_{0 a}(n)$ and $h_{0 b}(n)$ represent low-pass filters whereas $h_{1 a}(n)$ and $h_{1 b}(n)$ represent high-pass filters for trees $a$ and $b$. Filter sets at the first level are different from filter sets at level $j>1$. Details about filters design for the dual-tree $\mathbb{C W T}$ can be found in [7].

Approximation coefficients are obtained from real low-pass subband at the final level of the forward transform. In the case of details coefficients, they are obtained from complex high-pass subband at each level of the forward transform. The frequency content analysis is controlled by the scale factor $j$.

It is noteworthy that each DWT is composed of two different sets of filters satisfying the perfect reconstruction condition. In this sense, the filter sets should be designed taking into account some additional requirements to the DWT in order to the overall transform be approximately analytic, or equivalently, $\psi_{i} \approx \mathcal{H}\left\{\psi_{r}\right\}[2]$.

In order to invert the dual-tree $\mathbb{C W T}$, the inverse transform of each of the two real DWTs are used. After obtaining these two signals, they are averaged to obtain the inverse transform [2].

From (9), algorithms based on complex wavelets can exploit the magnitude, (10), and phase, (11), of the complex wavelet coefficients:

$$
\begin{gathered}
\left|d_{c}(j, n)\right|=\sqrt{\left[d_{r}(j, n)\right]^{2}+\left[d_{i}(j, n)\right]^{2}}, \\
\angle d_{c}(j, n)=\arctan \left(\frac{d_{i}(j, n)}{d_{r}(j, n)}\right) .
\end{gathered}
$$

This approach is relatively recent and has been greatly explored by researchers in signal processing. For example, it may be mentioned the works in [10] and [11].

\section{Fault Identification in Aeronautical Structures using the Dual-Tree $\mathbb{C W T}$}

As it was previously mentioned, the performance of the dual-tree $\mathbb{C W T}$ in fault identification in aeronautical structures will be evaluated. Furthermore, comparisons with DWT results will be performed.

The proposed method consists in a multiscale analysis of the phase of the complex wavelet coefficients provided by dual-tree $\mathbb{C W T}$. Scales $j(j=1,2,3,4)$ are analyzed and the phase is obtained from equation (11). The phase is very sensitive to variations of the signal and can capture small flaws, as it can be seen in Figure 3. 
The proposed model to evaluate the methodology, obtained by finite element method, was an aluminum beam in the cantilever-free condition discretized with 10 finite elements with 2 degrees of liberty each. The material properties used are the modulus of elasticity $(E=700 \mathrm{GPa})$ and the density $\left(\gamma=2710 \mathrm{~kg} / \mathrm{m}^{3}\right)$. Dimensions are $500 \mathrm{~mm}$ long, $25 \mathrm{~mm}$ wide and $5 \mathrm{~mm}$ thick. Figure 2 illustrates the patterned beam [13]. From the beam model several simulations were performed with different locations of faults. Percentages of wear was fixed at $1 \%$ in order to check if the method identifies small flaws. The database consists of generated signals captured by an accelerometer attached to the beam. In all simulations the beam was excited in the 3rd degree of freedom and the signal was captured on the 19th degree of freedom. Therefore, simulations consisted in 550 signals in the structure, 500 without wear (base-line condition) and 50 with wear (structural failure). The wear failure was placed in five locations (finite elements 1, 3, 5, 8 and 10).

All results were obtained by using Antonini (9,7)-tap filters at first level and q-shift $(10,10)$-tap filters at level $j>1$ [7]. In order to compute the conventional DWT, Daubechies length 10 filters were used.

In Figure 3, for the base-line signal and the same with $1 \%$ of failure (a), follows the wavelet coefficients at scale $j=1$ (b) and (c). (b) real wavelet coefficients computed using a conventional DWT. (c) phase of the complex wavelet coefficients computed using a dual-tree $\mathbb{C W T}$.

In order to compare DWT and dual-tree $\mathbb{C W T}$ performances in fault identification, Pearson's correlation coefficient will be used. Pearson's correlation coefficient ranges in the interval $[-1,1]$, measuring the mutual relationship between base-line signal and the failure signal. Correlation coefficient equal to 1 or -1 implies a perfect correlation, whereas correlation coefficient equal to 0 means that the signals are not linear correlated [14]. For the purposes of this paper, as smaller is the correlation coefficient as better is the fault detection.

In time domain, the correlation between base-line condition signal and the same with $1 \%$ of failure at finite element 1 is 0.9987 . Table 1 shows the correlation between the same signals in the wavelet domain across scales $j(j=1,2,3,4)$.

Observing the results presented in Table 1, it is worth seeing that complex wavelets are better than real wavelets in small flaws identification. Furthermore, the fault was identified in scales $j(j=1,2,3)$.

The analysis of structural integrity was performed presenting the 550 signals randomly and checking, from phase, which are flawed. Using an simple 
Table 1: Performance comparisons between DWT and dual-tree $\mathbb{C W T}$ in small flaws identification.

\begin{tabular}{c|c|c}
\hline \multicolumn{3}{c}{ correlation index } \\
\hline Scale & conventional DWT & dual-tree $\mathbb{C W T}$ \\
\hline 1 & 0.9928 & 0.7555 \\
2 & 0.9985 & 0.7961 \\
3 & 0.9994 & 0.8058 \\
4 & 0.9998 & 0.9259 \\
\hline
\end{tabular}

threshold, empirically defined from Pearson's correlation coefficient, it is found a success rate of $100 \%$.

In addition to fault identifying, the magnitude acquired from (10) can provides information about the intensity of failure. Figure 4 shows the magnitude of the complex wavelet coefficients at scale $j=1$ for the base-line signal and the same with $1 \%$ and $5 \%$ of failure at finite element 5 .

Analyzing Figure 4, it is found that $\left|d_{c}(j, n)\right|$ ranges according to the fault level of the signal, unlike the phase which is uncorrelated with the energy of the signal in time domain.

\section{Conclusion}

In this paper, the application of dual-tree $\mathbb{C W T}$ on fault monitoring and identification in aeronautical structures has been proposed. The dual-tree $\mathbb{C W T}$ is nearly shift-invariant and directionally selective in two and higher dimensions, being considered a relatively recent improvement for the conventional DWT.

The phase of the complex wavelet coefficients is very sensitive to variations of the signal and capture small flaws, whereas the magnitude provides information about the intensity of failure. For the application studied, the dual-tree $\mathbb{C W T}$ showed to be a powerful tool to fault identification, overcoming the real DWT. Simulation results showed that the proposed method presented good results, obtaining a hit rate of $100 \%$. In simulations, there were used signals with $1 \%$ of failure, in different locations, in order to check the performance of method on small flaws.

For further works, magnitude and phase of the complex wavelet coefficients could be used as input signals to neural networks or artificial immune systems for the development of the more elaborate systems. 
96 C.C.E. Abreu, F.R. Chavarette, F. Villarreal, M.A.Q. Duarte, F.P.A. Lima

\section{Acknowledgments}

Authors would like to thank Brazilian agencies CAPES (PhD grant) and CNPq (Proc. n. 301769/2012-5).

\section{References}

[1] I. Daubechies, Ten Lectures on Wavelets, SIAM, USA, (1992). [DOI: http://dx.doi.org/10.1137/1.9781611970104]

[2] I. W. Selesnick, R. G. Baraniuk, N. G. Kingsbury, The dual-tree complex wavelet transform-A coherent framework for multiscale signal and image processing, IEEE Signal Processing Magazine, 22 (2005), 123-151. [DOI:10.1109/MSP.2005.1550194]

[3] J. M. Lina, M. Mayrand, Parametrizations for Daubechies wavelets, Phys. Rev. E, 48 (1993), R4160-R4163. [DOI:10.1103/PhysRevE.48.R4160]

[4] S. Mallat, A wavelet tour of signal processing, Academic Press, USA, (1998).

[5] H. M. Paiva, M. N. Martins, R. K. H. Galvo, J. P. L. M. Paiva, On the Space of Orthonormal Wavelets: Additional Constraints to Ensure Two Vanishing Moments, Signal Processing Letters, IEEE, 16 (2005), 101-104. [DOI:10.1109/LSP.2008.2009836]

[6] N. G. Kingsbury, The dual-tree complex wavelet transform: A new technique for shift invariance and directional filters, Proceedings 8th IEEE DSP Workshop, Utah, (1998), 9-12.

[7] N. G. Kingsbury, Design of q-shift complex wavelets for image processing using frequency domain energy minimization, Proceedings of the IEEE International Conference on Image Processing, Barcelona, 1 (2003), 1013-1016. [DOI:10.1109/ICIP.2003.1247137]

[8] G. Strang, T. Nguyen, Wavelets and filter banks, Wellesley-Cambridge Press, EUA, (1996).

[9] A. V. Oppenheim, R. W. Schafer,Discrete-time signal processing, Prentice Hall, Englewood Cliffs, USA, (1989). 
[10] A. Das, M. Bhuiyan, S. Alam, Statistical parameters in the dual tree complex wavelet transform domain for the detection of epilepsy and seizure, Electrical Information and Communication Technology (EICT), 2013 International Conference on, (2014), 1-6. [DOI:10.1109/EICT.2014.6777821]

[11] A. Khare, M. Khare, R. Srivastava, Dual tree complex wavelet transform based multiclass object classification. Machine Learning and Applications (ICMLA), 2013 12th International Conference on, 2 (2013), 501-506. [DOI: 10.1109/ICMLA.2013.167]

[12] T. Weickert, C. Benjaminsen, U. Kiencke, Analytic complex wavelet packets for speech enhancement. IEE International Conference on Acoustic, Speech, and Signal Processing, (ICASSP 2008), 1 (2008), 3269-3272. [DOI:10.1109/ICASSP.2008.4518348]

[13] F. P. A. Lima, F. R. Chavarette, A. S. Souza, S. S. F. Souza, M. L. M. Lopes, Artificial Immune Systems with Negative Selection Applied to Health Monitoring of Aeronautical Structures. Advanced Materials Research, 871 (2013), 283-289.

[DOI:10.4028/www.scientific.net/AMR.871.283]

[14] A. Field, Discovering Statistics Using SPSS, Sage, England, (2005). 


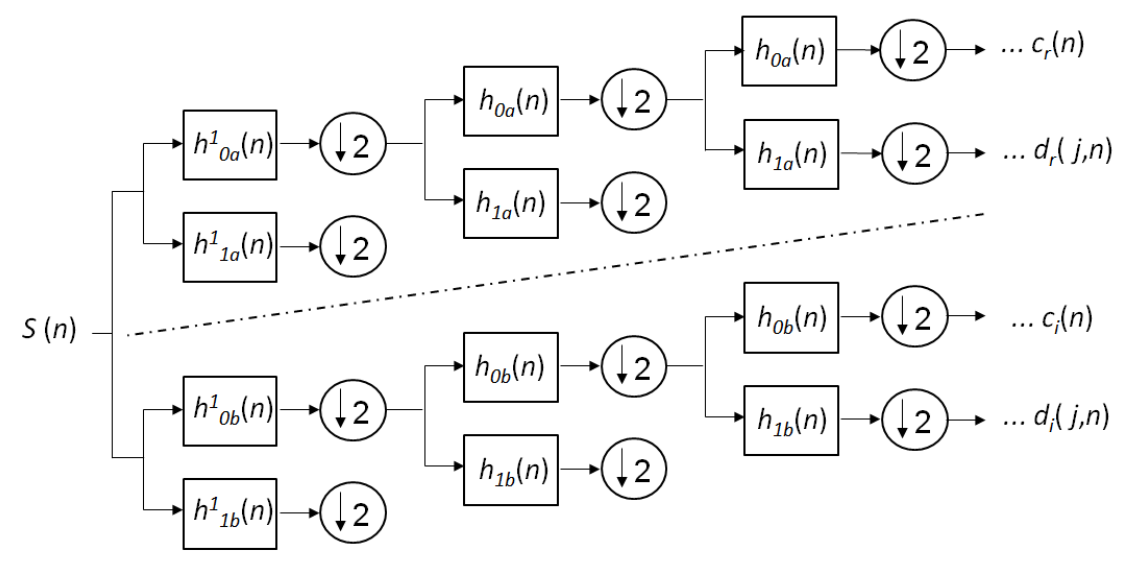

Figure 1: Filter bank analysis for the dual-tree $\mathbb{C W T}$.

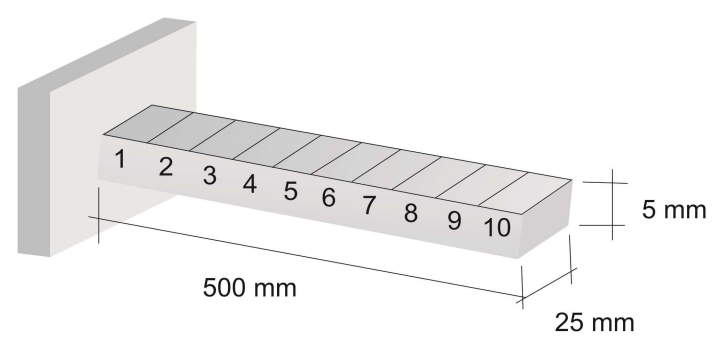

Figure 2: Beam modeled. 

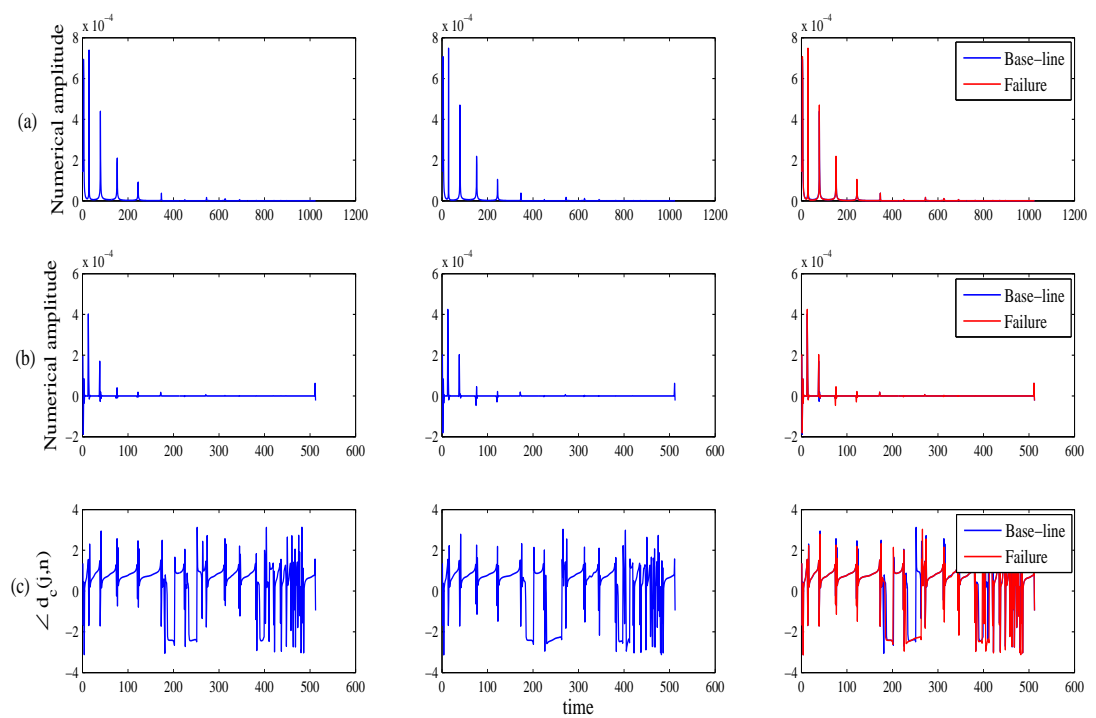

Figure 3: Fault identification performance between DWT and dual-tree $\mathbb{C W T}$.
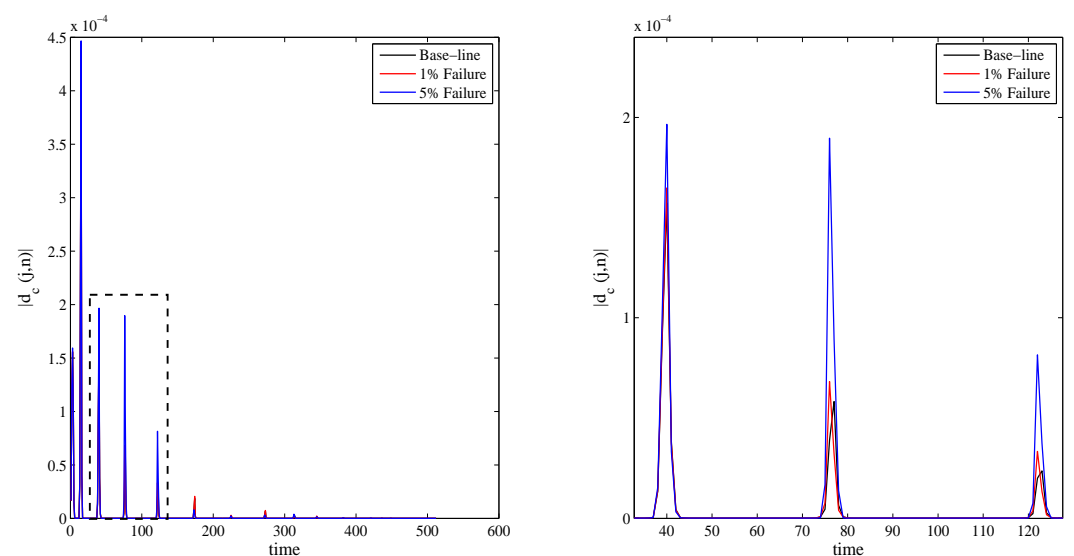

Figure 4: Fault level identification from $\left|d_{c}(j, n)\right|$. 